\begin{tabular}{|c|c|}
\hline Title & Some experimental studies on time of-flight radiography using a pulsed neutron source \\
\hline Author(s) & $\begin{array}{l}\text { Kiyanagi, Y.; Sakamoto, N.; I wasa, H.; Kamiyama, T.; Hiraga, F.; Sato, S.; Sagehashi, H.; Ino, T.; Furusaka, M.; } \\
\text { Suzuki, J.; Gorin, A.; Manuilov, I.; Ryazantsev, A.; Kuroda, K.; Sakai, K.; Tokanai, F.; Miyasaka, H.; A dachi, T.; Oku, } \\
\text { T.; Ikeda, K.; Suzuki, S.; Morimoto, K.; Shimizu, H.M. }\end{array}$ \\
\hline Citation & $\begin{array}{l}\text { IEEE Transactions on Nuclear Science, 52(1), 371-374 } \\
\text { https://doi.org/10.1109/ NS.2005.844299 }\end{array}$ \\
\hline Issue Date & 2005-02 \\
\hline Doc URL & http:/hdl.handle.net/2115/5700 \\
\hline Rights & $\begin{array}{l}\text { (O2005 IEEE. Personal use of this material is permitted. However, permission to reprint/republish this material for } \\
\text { advertising or promotional purposes or for creating new collective works for resale or redistribution to servers or lists, } \\
\text { or to reuse any copyrighted component of this work in other works must be obtained from the IEEE." } \\
\text { IEEEE, IEEE Transactions on Nuclear Science } \\
, 52,1,2005, \text { p371-374 }\end{array}$ \\
\hline Type & article \\
\hline File Information & ITONS52-1.pdf \\
\hline
\end{tabular}

Instructions for use 


\section{Some Experimental Studies on Time-of-Flight Radiography Using a Pulsed Neutron Source}

Y. Kiyanagi, N. Sakamoto, H. Iwasa, T. Kamiyama, F. Hiraga, S. Sato, H. Sagehashi, T. Ino, M. Furusaka, J. Suzuki, A. Gorin, I. Manuilov, A. Ryazantsev, K. Kuroda, K. Sakai, F. Tokanai, H. Miyasaka, T. Adachi, T. Oku, K. Ikeda, S. Suzuki, K. Morimoto, and H. M. Shimizu

\begin{abstract}
We are developing a fiber type two-dimentional (2-D) position-sensitive detector having a spatial resolution less than $0.5 \mathrm{~mm}$ in order to use it in the time-of-flight (TOF) measurements. The detector performance was examined, and some experiments on the TOF radiography were performed using this detector. The results indicated that the detector could be applied successfully to the TOF measurement and that the TOF radiography was very useful since it gave more informative data than those obtained by the conventional radiography using a broad energy band.
\end{abstract}

Index Terms-Bragg cutoff, cold neutron, fiber type detector, material identification, spatial resolution, time-of-flight.

\section{INTRODUCTION}

$\mathbf{N}$ EUTRON radiography using a pulsed neutron source is not very popular since the pulsed neutron sources afforded for such measurements are scarce. By using the time-of-flight (TOF) method combined with the pulsed neutron sources, we can get different contrast images at once, depending on the energy and neutron cross sections of objectives. The cross section data will be used for the distinction of materials in the objectives and may also give information concerning the deformation of the crystal lattice. Such a possibility will increase the usefulness of the radiography.

Now, a high-intensity pulsed spallation neutron source is under construction in Japan as well as in the United States. The pulsed spallation neutron sources will become major neutron sources since building the new research reactors is very difficult at present. Therefore, radiography using a pulsed neutron source is now in a stage to be developed intensively. We can use the pulsed neutron source as a reactor. However, it is much better to use the pulse nature effectively.

Manuscript received September 15, 2002; revised August 15, 2004.

Y. Kiyanagi, N. Sakamoto, H. Iwasa, T. Kamiyama, and F. Hiraga are with the Graduate School of Engineering, Hokkaido University, Sapporo 060-8628, Japan (e-mail kiyanagi@qe.eng.hokudai.ac.jp).

S. Sato, H. Sagehashi, T. Ino, and M. Furusaka are with the High Energy Accelerator Research Organization, Tsukuba, Japan.

J. Suzuki is with the Japan Atomic Energy Research Institute, Tokai, Ibaraki, Japan.

A. Gorin, I. Manuilov, and A. Ryazantsev are with the Institute for High Energy Physics, Protvino, Moscow region, Russia.

K. Kuroda is with the Advanced Research Institute for Science and Engineering, Waseda University, Tokyo, Japan.

K. Sakai is with the Department of Physics, Tokyo Institute of Technology, Tokyo, Japan

F. Tokanai is with the Department of Physics, Yamagata University, Yamagata, Yamagata, Japan.

H. Miyasaka, T. Adachi, T. Oku, K. Ikeda, S. Suzuki, K. Morimoto, and H. M. Shimizu are with the RIKEN, Wako, Saitama, Japan.

Digital Object Identifier 10.1109/TNS.2005.844299

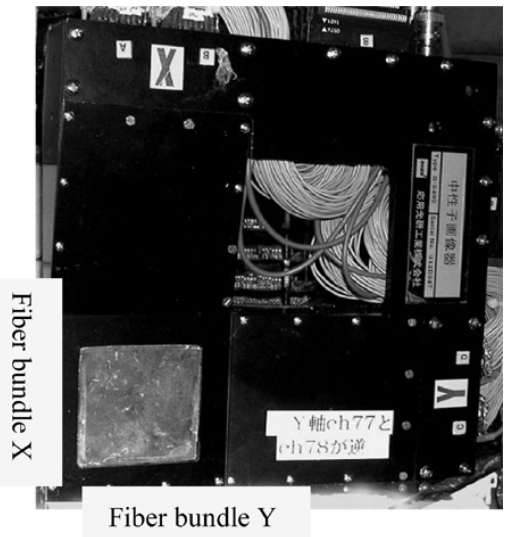

Fig. 1. Photo of the fiber type 2-D position-sensitive detector.

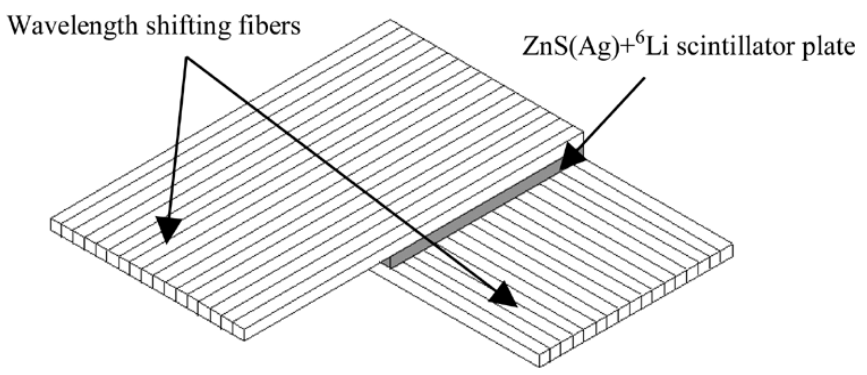

Fig. 2. Schematic view of the detector censer part indicating simply principle.

If we intend to use the TOF method for the radiography, we need a detector that has a good spatial resolution and a short time resolution. There are He gas-type detectors (microstrip gas chamber and microwire gamma camera), but the spatial resolution is usually about few millimeters, although they can be used for the TOF measurements. On the other hand, an imaging plate has a good spatial resolution, but the TOF measurement is impossible. Two-dimensional (2-D) position-sensitive detectors with high spatial resolution have been developed [1]. We are also developing a fiber-type 2-D position-sensitive neutron detector for evaluating neutron optical devices [2], [3], which consists of 0.4-mm fiber arrays, giving a resolution about this size. We have developed electronics for the TOF measurement for this detector and tried to apply this detector to the TOF radiography. Here, we mention the outline of performance of the detector and some images obtained by the TOF radiography to demonstrate the effectiveness. 


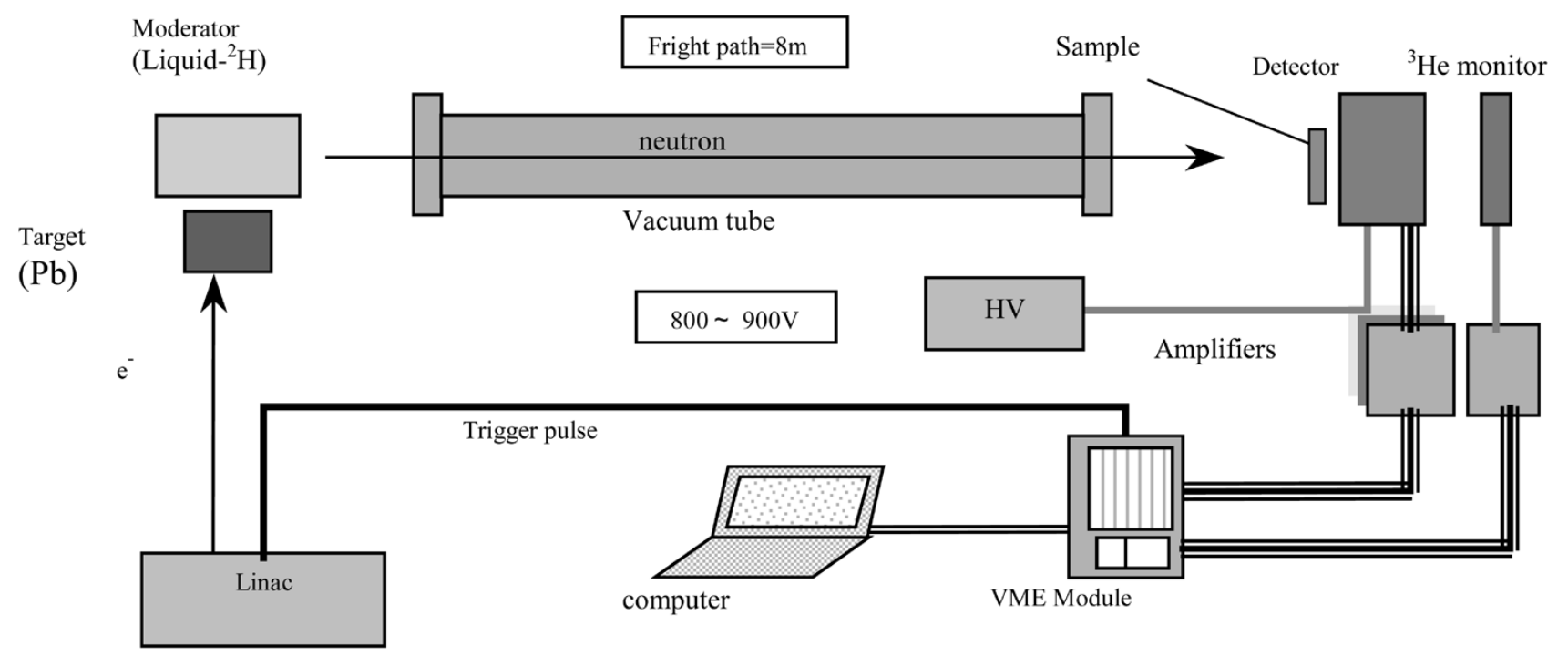

Fig. 3. Experimental setup for the detector check and the radiography.

\section{EXPERIMENTAL}

The detector consists of the detection area with a size of about $60 \times 60 \mathrm{~mm}$, two fiber arrays connecting to the photo multipliers, and four photo multipliers. The photo is shown in Fig. 1. The detection area is in the lower left region, and a thin $(0.3-\mathrm{mm}) \mathrm{Al}$ input window is placed in front of the detection area to minimize neutron scattering, spoiling the space resolution. The wavelength shifting (WLS) fibers are on the left and lower right parts in black.

The structure of the detection area is schematically shown in Fig. 2. The detection part is a thin $(0.4 \mathrm{~mm}) \mathrm{ZnS}(\mathrm{Ag})+{ }^{6} \mathrm{Li}$ scintillator plate that is $58 * 58 \mathrm{~mm}^{2}$ in size. The scintillator is semitransparent, and it emits light in the region of $\lambda-430 \mathrm{~nm}$ with a fast decay constant of $-200 \mathrm{~ns}$. The overall decay time is on the order of tens of microseconds.

For the light readout, two fiber arrays (with 128 WLS fibers each) were used. Fibers are $-150 \mathrm{~mm}$ long, and the crosssection is $0.4 * 0.4 \mathrm{~mm}^{2}$. The far ends were attached to an $\mathrm{Al}$ mirror. The fibers are alternated with reflectors $(50-\mu \mathrm{m}$-thick aluminized Mylar) to prevent the optical cross-talk between fibers, and the resulting fiber pitch was $0.45 \mathrm{~mm}$. The arrays were glued with an optical epoxy on the top (X-layer) and bottom (Y-layer) of the scintillator in orthogonal directions to supply the 2-D readout.

The WLS fibers were optically connected to the 64-channel multianode photo multiplier (PSPM). The detector has four tubes: two for the X- and two for the Y-projection. The electronic pulses from the PSPM are transferred to the amplifier modules. The amplifier modules were composed of eight plates that consisted of 32 amplifier chips. The amplified pulses are transferred to Analog Digital Converters (ADC) through a discriminator to eliminate the noise. A pair of digital pulses from the X-fiber and the Y-fiber that are detected at the same time are collected and arranged by master module to make the 2-D image. The process needs 2 to $5 \mu \mathrm{s}$.

Then, to perform TOF measurements, we made a module. This system has a maximum of 256 time channels, and the width of a time channel can be set larger than $1 \mu$ s although usually, the width of the time bin is about a maximum of $80 \mu$ s since the repetition rate is about $50 \mathrm{~Hz}$. As a kicker determining the time origin, a trigger pulse of the linac was supplied to the system of the TOF measurement.

The master and the ADC modules were assembled in a standard VME, and the programs to control the modules were written with the graphical program software LabVIEW4.0.

The experiments were performed at the electron linear accelerator (linac) facility at Hokkaido University. The linac at Hokkaido University is a unique pulsed-neutron facility used for the various neutron experiments in which a cold neutron source or a thermal neutron source can be chosen; in addition, the linac power can be adjusted, depending on the demand of the experiments. The experimental setup for the present experiment is shown in Fig. 3. A liquid-hydrogen cold-moderator at $18 \mathrm{~K}$ was used since the differences in the contrast, as well as in the cross section, strongly appear in the cold neutron region. The detector developed was located $8 \mathrm{~m}$ from the moderator. A vacuum flight tube with a length of $5 \mathrm{~m}$ was placed between the moderator and the detector. $\mathrm{A}^{3} \mathrm{He}$ detector was also used as a monitor.

\section{Performance of the Detector}

We measured the transmission of a $\mathrm{Cd}$ sheet having $0.5 \mathrm{~mm}$ square holes every $5 \mathrm{~mm}$ along $\mathrm{X}$ and $\mathrm{Y}$ directions. Fig. 4 shows an image of the transmission data, indicating the spatial resolution. The spatial resolution is roughly estimated to be less than $0.5 \mathrm{~mm}$. We also measured the transmission of a $\mathrm{Cd}$ sheet with $0.5-\mathrm{mm}$ line holes along the $\mathrm{X}$ or $\mathrm{Y}$ directions. The intensity distribution along the $X$ direction is plotted in Fig. 5. Fitting the Gauss function to the peak showed that the full width at half maximum was less than $0.4 \mathrm{~mm}$. It should be noted that the Gauss fit is not very good for such a rectangle-like distribution. However, the results indicate that the detector has a good resolution of approximately less than $0.5 \mathrm{~mm}$. This resolution is the highest one among the detectors that are applicable to the TOF method.

However, the efficiency of this detector will be not very high since the efficiency of the scintillator and the capture of light in 


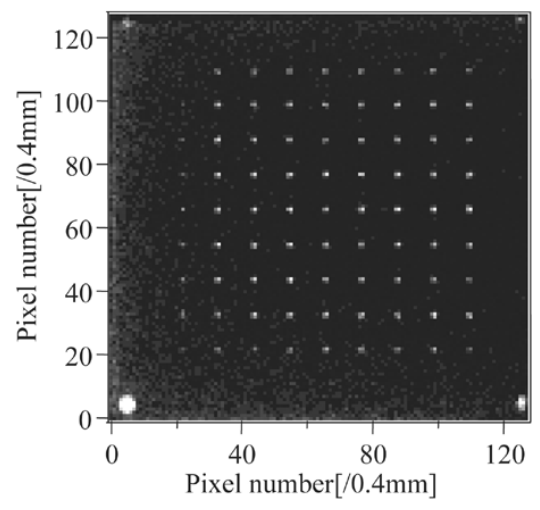

Fig. 4. Image indicating the spatial resolution.

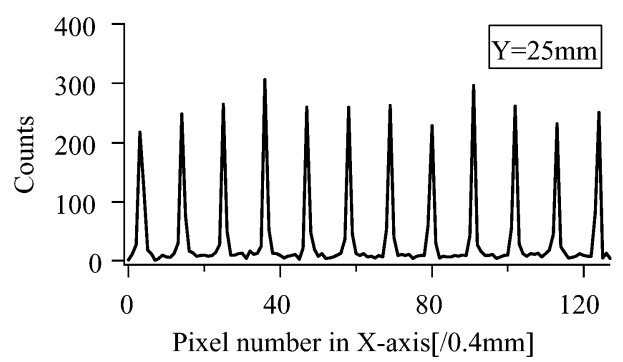

Fig. 5. One-dimensional distribution.

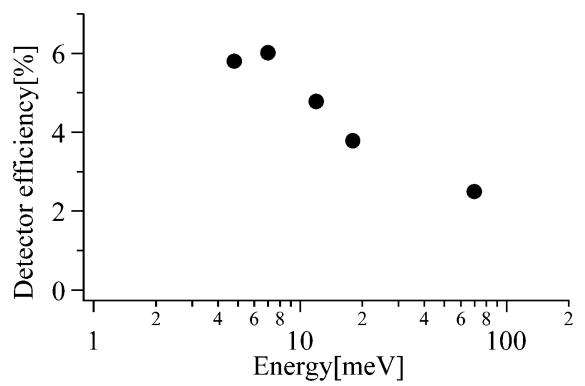

Fig. 6. Detector efficiency.

the fibers is not very high. We checked the detector efficiency by comparing the efficiency of the He-3 detector. Fig. 6 shows the detector efficiency. As expected, the efficiency decreases by increasing the energy. The efficiency at the thermal neutron region is about 5 to $6 \%$ and, at $100 \mathrm{meV}$, about $2 \%$. This is a shortcoming of the detector that will be improved in the future.

We also examined the maximum counting rate of this detector. The rate is about 10000 counts/s, and over this count rate, the image showed a grid pattern. We need to look for some method to improve the maximum count rate. We were also interested in the noise level of the detector, which is probably due to gamma rays, since our linac is also an intense gamma ray source. The effect of the noise was slightly higher at the higher energy region-namely, the short time region-which is due to the gamma flash emitted at the instance when an electron burst hit the target. However, even in such a region, the level was about $4 \%$, and the rate was almost 1 to $2 \%$ over the whole energy region-namely, over the whole measured time span. The noise level is low enough to perform the precise experiments.

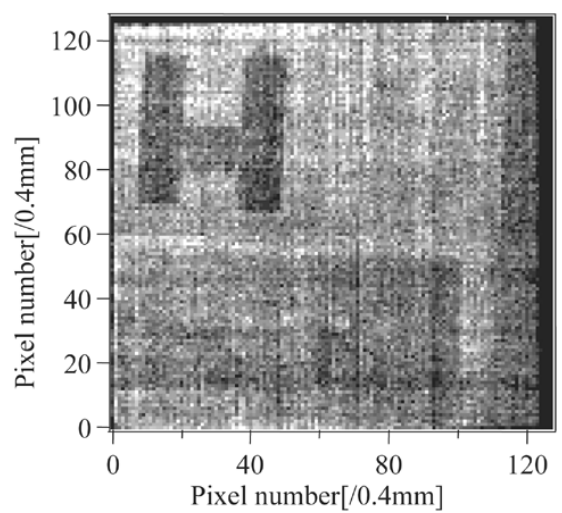

(a)

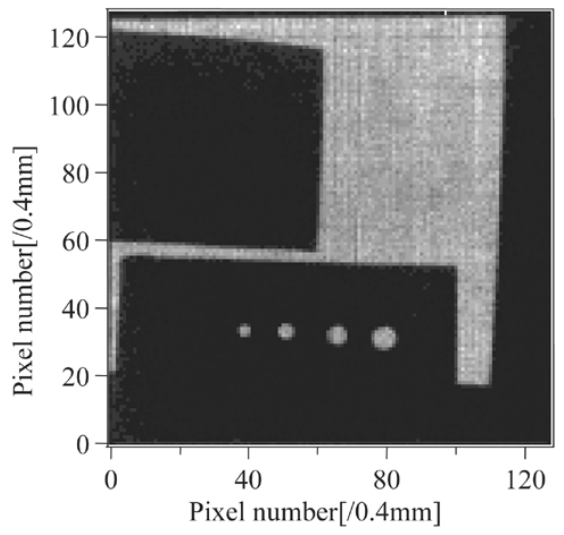

(b)

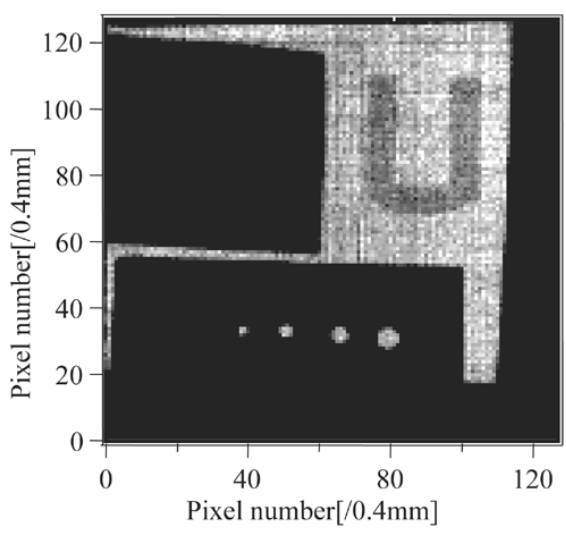

(c)

Fig. 7. (a) Images obtained at different energies $(\mathrm{En}=0.47-1.7 \mathrm{eV})$ (b) Images obtained at different energies $(\mathrm{En}=0.075-0.17 \mathrm{eV})$. (c) Images obtained at different energies $(\mathrm{En}=0.0015-0.0017 \mathrm{eV})$.

\section{IMAGING USING THE TOF METHOD}

One of the unique features of the TOF imaging is that we can obtain the different contrast images at once, which enables us to distinguish the various materials in the object. As an example, we measured images shown in Fig. 7. A character " $\mathrm{H}$ " made with 5 -mm polyethylene was covered by a $\mathrm{Cd}$ sheet, and a character "U" was made with thin vinyl. The results are also shown in Fig. 7(a)-(c). In Fig. 7(a), we can see the character "H" under the $\mathrm{Cd}$ since the energy range analyzed was higher than the $\mathrm{Cd}$ cut-off energy, while the character "U" cannot be seen clearly since it is too thin. At a medium energy range, both $\mathrm{H}$ and $\mathrm{U}$ 


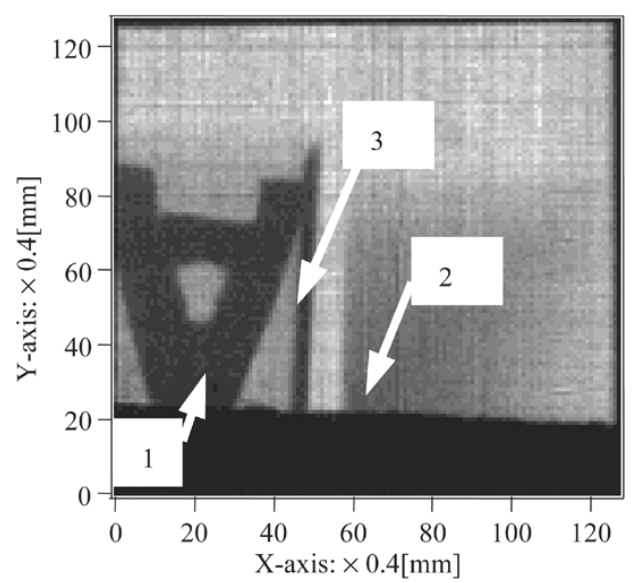

(a)

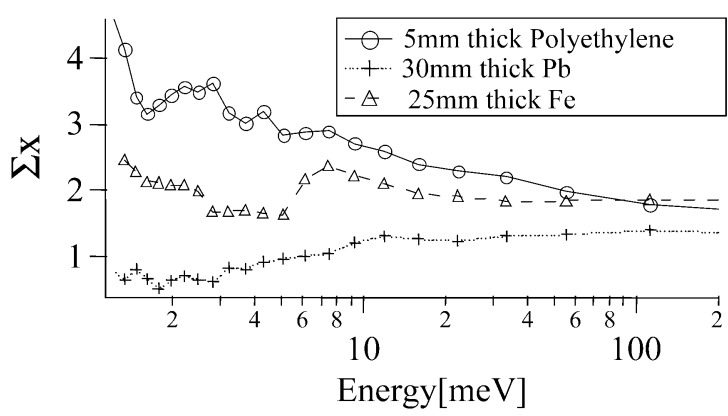

(b)

Fig. 8. (a) Image and $\Sigma x$ of each material (image of different materials). (b) Image and $\Sigma x$ of each material ( $\Sigma x$ at different areas).

cannot be seen, as shown in Fig. 7(b). At low energy, the character " $U$ " is seen, and naturally, " $H$ " cannot be seen due to the $\mathrm{Cd}$ sheet on it.

We also have an image between these energies, and at that energy, both characters were not seen. Therefore, in order to get images at different energies at once, it is very useful not to miss the materials that exist in the object.

We can get more detailed information by inspecting the energy-dependent feature of the transmission. Furthermore, we will be able to identify the material in the object by analyzing the total neutron cross section data of the materials if they have characteristic features in the low-energy region, such as the Bragg cutoff. Fig. 8(a) shows an image obtained by the TOF method. A character " $A$ " is seen clearly, and at the right side of the character, a black rectangular image exists; furthermore, a shadow-like black image appears on the right side of the rectangular image. To distinguish these materials, we obtained data of the macro cross section $(\Sigma)$ times the thickness $(x)$ at the positions indicated in the figure by numbers 1,2 , and 3 [Fig. 8(b)]. The $\Sigma x$ at position 1 increases with decreasing energy, which corresponds to the feature of the material having a large absorption cross section or a large incoherent cross section, although there are some dips in the data. The data at position $2 \Sigma x$ decreases as the energy decrease, which is an unusual change in the cross section, and the data at position 3 show dips due to the Bragg cutoff. We can recognize from these results that at least they are different materials. The material at position 1 is polyethylene in an iron box, that at position 2 is lead, and that at position 3 is iron. The data for polyethylene has some dips at the low-energy region, but this may be due to poor statistics, although a dip would appear that corresponds to the iron Bragg cutoff. The cross section feature at position 2 is typical for lead since lead has no clear Bragg cutoff. The data at position 3, of course, show the Bragg cutoff at the energy corresponding to the iron data in the BNL-325. We also deduced the macro cross section from these data, and they corresponded to the value of each of the materials. Therefore, if we can observe the change of the cross section at low energy, we will identify the materials.

\section{CONCLUSION}

The detector based on the fiber-scintillator is very useful for the neutron radiography using the TOF method. The results obtained by using this detector illustrated the usefulness of getting the different contrast data at once as well as the possibility to identify the materials in the object. Although we need more improvement, it is found that such radiography will give more informative data than the one obtained by a conventional method.

\section{REFERENCES}

[1] G. Bruckner, A. Czermak, H. Rauch, and P. Weilhammer, Nuclear Instrum. Methods, vol. A424, pp. 183-189, 1999.

[2] K. Kuroda and I. Manuilov, Nuclear Instrum. Methods, vol. A430, pp. 311-320, 1999.

[3] A. Gorin, K. Kuroda, I. Manuilov, K. Morimoto, T. Oku, A. Ryazantsev, H. M. Shimizu, J. Suzuki, and F. Tokanai, Nuclear Instrum. Methods, vol. A479, pp. 456-460, 2002. 